\title{
Use of simple clinical and laboratory predictors to differentiate influenza from dengue and other febrile illnesses in the emergency room
}

\author{
Shi-Yu Huang ${ }^{1}$, Ing-Kit Lee ${ }^{2 *}$, Lin Wang ${ }^{3}$, Jien-Wei Liu², Shih-Chiang Hung ${ }^{1}$, Chien-Chih Chen ${ }^{1}$, Tzu-Yao Chang ${ }^{2}$
} and Wen-Chi Huang ${ }^{2}$

\begin{abstract}
Background: Clinical differentiation of influenza from dengue and other febrile illnesses (OFI) is difficult, and available rapid diagnostic tests have limited sensitivity.

Methods: We conducted a retrospective study to compare clinical and laboratory findings between (i) influenza and dengue and (ii) influenza and OFI.

Results: Of 849 enrolled patients, the mean time between illness onset and hospital presentation was 1.7, 3.7, and 3 days for influenza, dengue, and OFI, respectively. Among pediatric patients ( $\leq 18$ years) (445 influenza, 24 dengue, and $130 \mathrm{OFI}$ ), we identified absence of rashes, no leukopenia, and no marked thrombocytopenia (platelet counts $<100 \times 10^{9}$ cells/L) as predictors to distinguish influenza from dengue, whereas rhinorrhea, malaise, sore throat, and mild thrombocytopenia (platelet counts $100-149 \times 10^{9} / \mathrm{L}$ ) were predictors that differentiated influenza from OFI. Among adults (>18 years) (81 influenza, 124 dengue, and $45 \mathrm{OFI}$ ), no leukopenia and no marked thrombocytopenia distinguished influenza from dengue, while rhinorrhea and malaise differentiated influenza from OFI. A diagnostic algorithm developed to distinguish influenza from dengue using rash, leukopenia, and marked thrombocytopenia showed $>90 \%$ sensitivity to identify influenza in pediatric patients.

Conclusions: This study identified simple clinical and laboratory parameters that can assist clinicians to distinguish influenza from dengue and OFI. These findings may help clinicians diagnose influenza and facilitate appropriate management of affected patients, particularly in resource-poor settings.
\end{abstract}

Keywords: Influenza, Dengue, Other febrile illnesses

\section{Background}

Influenza is one of the most common infectious diseases worldwide [1]. The spectrum of clinical manifestations ranges from mild-form nonspecific febrile illness, such as cough, sore throat, headache, rhinorrhea, malaise, and muscle ache, to respiratory failure and death [2-4]. Its symptoms and signs can be similar to other viral illnesses such as dengue, making the infections difficult to distinguish. The clinical manifestations of dengue illness vary greatly, ranging from a mild, flu-like, and selflimited febrile illness to severe dengue [5,6]. Influenza

\footnotetext{
* Correspondence: leee@cgmh.org.tw

${ }^{2}$ Division of Infectious Diseases, Department of Internal Medicine, Kaohsiung Chang Gung Memorial Hospital, Kaohsiung 833, Taiwan

Full list of author information is available at the end of the article
}

and dengue overlap geographically in tropical and subtropical regions of the world [1,7]. Concurrent influenza and dengue outbreaks have been reported [8-11]. The overlapping clinical features of influenza and dengue created clinical diagnosis and management challenges during simultaneous influenza and dengue outbreaks in Puerto Rico in 1977 [11]. Dengue epidemics have occurred in Taiwan for decades [12,13]. An outbreak of 2009 pandemic H1N1 occurred in Taiwan after the first case was identified on May 20, 2009 [14]. The overlapping symptoms and signs of influenza and dengue created a confusing clinical situation and challenges in etiology identification. Although rapid diagnostic tests can help confirm an influenza diagnosis, their sensitivity ranges from $40-70 \%$ depending on the day of illness and specimen type [15]. Definitive 
diagnosis of dengue is made using serology tests, but these tests are not always readily available in most clinical laboratories $[7,16]$. Our study aimed to identify clinical and laboratory features that distinguish influenza from dengue and other febrile illnesses (OFI) in dengue and non-dengue endemic settings. We applied decision tree analysis to our dataset to discriminate patients with influenza from those with dengue. Our findings may be valuable for clinicians working in crowded emergency rooms (ER) in countries with limited medical resources.

\section{Methods}

\section{Ethics statement}

The study was approved by the Institutional Review Board of Kaohsiung Chang Gung Memorial Hospital (KSCGMH) (Document no. 102-4695B). Informed consent was not required as the data were analyzed anonymously.

\section{Study population, diagnosis and definition}

We conducted a retrospective study of 849 febrile patients (ear temperature $\geq 38^{\circ} \mathrm{C}$ ) who presented during 2008 and 2010 with possible dengue and influenza infections to the ER at KSCGMH, a 2,600-bed primary care and tertiary referral medical center in southern Taiwan. All patient medical records were reviewed, and clinical and laboratory data at the time of hospital presentation were extracted for analysis.

Patients with influenza infections were defined as those laboratory-positive for influenza with influenza-like illness. Influenza-like illness was defined according to World Health Organization (WHO) guidelines: fever, cough, or sore throat [17]. Laboratory diagnosis of influenza was made for patient's respiratory specimens (nasopharyngeal or pharyngeal swabs) positive for virus-specific ribonucleic acid by real-time reverse transcriptase-polymerase chain reaction (RT-PCR) (TAIGEN Bioscience Corporation, Taiwan) [18]. All PCRs were performed at the central testing laboratory of KSCGMH using the standard real-time RT-PCR influenza procedure described by the Centers for Disease Control and Prevention [19].

All dengue cases included in this study were confirmed by at least one of the following criteria: (i) positive dengue virus-specific real-time RT-PCR (QuantiTect SYBR Green RT-PCR kit; Qiagen, Hilden, Germany) [20], (ii) positive dengue virus-specific immunoglobulin (Ig) $\mathrm{M}$ antibody enzyme-linked immunosorbent assay of acute-phase serum, after excluding cross-reactions to Japanese encephalitis virus [21], (iii) a fourfold increase in dengue virus-specific IgG antibody in convalescent serum compared to the acute phase, and (iv) acutephase serum positive for dengue virus-specific nonstructural glycoprotein-1 (NS1) antigen (Bio-Rad Laboratories, Marnes-la-Coquette, France) [22,23]. Dengue diagnostic tests were performed by the Centers for Disease Control and Prevention in Taiwan. Dengue patients in this study were classified as dengue fever without warning signs, dengue fever with warning signs and severe dengue based on 2009 WHO case definitions [7]. Patients who did not meet the laboratory diagnosis criteria for influenza and dengue were classified as OFI in our analyses.

Leukopenia was defined as a peripheral white cell count $<3.0 \times 10^{9}$ cells $/ \mathrm{L}$ (reference value, 3.0-10.5 $\times 10^{9}$ cells/L), mild thrombocytopenia as a peripheral platelet count of $149-100 \times 10^{9}$ cells/L, and marked thrombocytopenia as a peripheral platelet count $<100 \times 10^{9}$ cells/L. Acute hepatitis referred to serum alanine aminotransferase (ALT) levels greater than 1,000 U/L (reference value, $<40 \mathrm{U} / \mathrm{L})$.

\section{Statistical analysis}

Patients were categorized as having (i) influenza, (ii) dengue, or (iii) OFI. To distinguish influenza from dengue or OFI in a dengue and non-dengue endemic settings, univariate analyses were performed to compare clinical and laboratory characteristics between (i) influenza and dengue, and (ii) influenza and OFI. Categorical variables were compared using Chi-square or Fisher exact tests, and continuous variables were compared using Student's $t$ or Mann-Whitney U tests. A 2-tailed P $<0.05$ was considered statistically significant. Significant variables in the univariate analyses were entered into a multivariate logistic regression model to determine independent predictor(s) of (i) influenza versus dengue, and (ii) influenza versus OFI. The sensitivity and specificity of each significant variable was assessed. Classification and regression tree analysis was performed [24]. The variables found to be significant in multivariate logistic regression with sensitivity greater than $80 \%$ were used to establish diagnostic decision trees to distinguish between patients with influenza and those with dengue [24]. Diagnosis was achieved by stepwise binary splitting, where one variable was entered at each node and, depending on the answer, a branch of the tree containing another variable was followed. Splitting stopped at nodes with a minimum classification of dengue, maximum classification of influenza, or a small number of patients in the node. For each terminal node, patients were classified as having low or high probability for influenza infection. To investigate the impact of age on the clinical presentation of influenza, dengue, and OFI, stratified analyses were performed for pediatric ( $\leq 18$ years) and adult ( $>18$ years) patients to examine the differences in clinical and laboratory characteristics between (i) influenza and dengue, and (ii) influenza and OFI.

\section{Results}

Demographic and clinical features of the patients Of 849 patients, 526 (315 men and 211 women; mean age, $13.6 \pm 13.5$ years) were diagnosed with influenza, 
148 (83 men and 65 women; mean age, $45.1 \pm 4.5$ years) with dengue, and 175 (106 men and 69 women; mean age, $15.3 \pm 15.9$ years) with OFI. None of the patients had concurrent influenza and dengue infections. Demographic, clinical, and laboratory information are summarized in Tables 1 and 2 .

Of 526 (445 [84.6\%] patients aged $\leq 18$ years) influenza infections, 447 (85\%) were 2009 pandemic H1N1 and 79 (15\%) were seasonal H3N2. The mean interval between onset of illness and the patient's arrival at the ER was $1.7 \pm 1.2$ days, and the mean fever duration was $2.8 \pm 1.6$ days. Besides fever, the 3 most common symptoms were cough $(67.6 \%)$, rhinorrhea $(63.3 \%)$, and sore throat $(39.3 \%)$. Mild thrombocytopenia was detected in 38 (15.9\%) of the 238 patients with an available peripheral platelet count. Of 364 patients receiving oseltamivir, $107(29.4 \%)$ started therapy $>48 \mathrm{~h}$ after illness onset. The
3 most common influenza-associated complications were pneumonia (5.1\%), meningoencephalitis (1.1\%), and acute respiratory distress syndrome (ARDS) (0.9\%) (Table 3). The mortality rate was $0.9 \%$, comprised of 5 patients (2 pandemic $2009 \mathrm{H} 1 \mathrm{~N} 1$ and 3 seasonal H3N2; 4 women and 1 man, median age of 24 years [range $2-72$ years]). All fatal cases received oseltamivir therapy $>48 \mathrm{~h}$ after illness onset. Pneumonia was detected in all 5 fatal cases, ARDS and meningoencephalitis each in 3 (60\% each), intracranial hemorrhage in 2 (40\%), and myocarditis and gastrointestinal bleeding each in 1 ( $20 \%$ each).

Among 148 patients diagnosed with dengue infection (24 [16.2\%] patients aged $\leq 18$ years), 64 (43.2\%) were dengue fever without warning signs, 77 (52\%) were dengue fever with warning signs, and $7(4.7 \%)$ were severe dengue, based on 2009 WHO case definitions [7]. An individual patient might have received more than one

Table 1 Demographics, clinical features, and outcomes of patients with influenza, dengue, and other febrile illnesses

\begin{tabular}{|c|c|c|c|c|c|}
\hline Variable & Influenza $(n=526)$ & Dengue $(n=148)$ & Other febrile illnesses $(n=175)$ & $P^{a}$ & $\mathbf{P}^{\mathbf{b}}$ \\
\hline Mean age $( \pm S D)$, years & $13.6 \pm 13.5$ & $45.1 \pm 4.5$ & $15.3 \pm 15.9$ & $<0.001$ & 0.941 \\
\hline \multicolumn{6}{|l|}{ Age group (n, [\%]) } \\
\hline$\leq 18$ years & 445 (84.6) & $24(16.2)$ & $130(74.3)$ & - & - \\
\hline$>18$ years & $81(15.4)$ & $124(83.8)$ & $45(25.7)$ & - & - \\
\hline Male gender (n, [\%]) & $315(59.9)$ & $83(56.1)$ & $106(60.6)$ & 0.406 & 0.873 \\
\hline \multicolumn{6}{|l|}{ Underlying condition ${ }^{c}(\mathrm{n},[\%])$} \\
\hline Bronchial asthma & $14(26.7)$ & 0 & $5(2.9)$ & - & - \\
\hline Diabetes mellitus & $10(1.9)$ & $17(11.5)$ & $2(1.1)$ & - & - \\
\hline Hypertension & $15(2.8)$ & $30(20.3)$ & $2(1.1)$ & - & - \\
\hline \multicolumn{6}{|l|}{ Influenza A virus subtype (n, [\%]) } \\
\hline Pandemic 2009 H1N1 & $447(85)$ & - & - & - & - \\
\hline Seasonal H3N2 & $79(15)$ & - & - & - & - \\
\hline \multicolumn{6}{|l|}{ Dengue disease severity (n, $[\%])$} \\
\hline Dengue without warning signs & - & $64(43.2)$ & - & - & - \\
\hline Dengue with warning signs & - & $77(52)$ & - & - & - \\
\hline Severe dengue & - & $7(4.7)$ & - & - & - \\
\hline \multicolumn{6}{|l|}{ Dengue virus serotypes (n/N, [\%]) } \\
\hline Serotype I & - & $10 / 40(25)$ & - & - & - \\
\hline Serotype $\|$ & - & $17 / 40(42.5)$ & - & - & - \\
\hline Serotype III & - & $12 / 40(30)$ & - & - & - \\
\hline Serotype IV & - & $1 / 40(2.5)$ & - & - & - \\
\hline $\begin{array}{l}\text { Mean interval from onset of symptoms to emergency } \\
\text { room presentation, days }( \pm S D)\end{array}$ & $1.7 \pm 1.2$ & $3.7 \pm 1.8$ & $3.0 \pm 2.1$ & - & - \\
\hline Mean fever duration, days ( \pm SD) & $2.8 \pm 1.6$ & $4.0 \pm 2.3$ & ND & $<0.001$ & - \\
\hline Use of oseltamivir >48 h (n/N, [\%]) & $107 / 364(29.4)$ & - & - & - & - \\
\hline Fatalities (n, [\%]) & $5(0.9)$ & 0 & 0 & 0.234 & 0.196 \\
\hline
\end{tabular}

$\mathrm{n} / \mathrm{N}=$ number of patients/total number of patients with data available; ND = no data.

${ }^{a}$ Influenza vs. dengue.

${ }^{b}$ Influenza vs. other febrile illnesses.

${ }^{\mathrm{C}} \mathrm{An}$ individual patient might have more than one underlying disease/condition. 
Table 2 Symptoms/signs and laboratory characteristics of patients with influenza, dengue, and other febrile illnesses

\begin{tabular}{|c|c|c|c|c|c|}
\hline Variable & Influenza $(n=526)$ & Dengue $(n=148)$ & Other febrile illnesses $(n=175)$ & $P^{a}$ & $\mathrm{P}^{\mathbf{b}}$ \\
\hline \multicolumn{6}{|l|}{ Symptom/sign ${ }^{c}$ (n [\%]) } \\
\hline Rhinorrhea & $333(63.3)$ & 0 & $73(41.7)$ & $<0.001$ & $<0.001$ \\
\hline Cough & $356(67.6)$ & $38(25.7)$ & $112(64)$ & $<0.001$ & 0.396 \\
\hline Sore throat & $207(39.3)$ & $24(16.2)$ & $43(24.6)$ & $<0.001$ & $<0.001$ \\
\hline Malaise & $183(34.8)$ & $95(64.2)$ & $21(12)$ & $<0.001$ & $<0.001$ \\
\hline Headache & $149(28.3)$ & $62(41.9)$ & $33(18.9)$ & 0.001 & 0.015 \\
\hline Vomiting/nausea & $116(22.1)$ & $43(29.1)$ & $37(21.1)$ & 0.076 & 0.801 \\
\hline Diarrhea & $57(10.8)$ & $15(10.1)$ & $26(14.9)$ & 0.807 & 0.154 \\
\hline Abdominal pain & $40(7.6)$ & $31(20.9)$ & $18(10.3)$ & $<0.001$ & 0.265 \\
\hline Joint pain & $26(4.9)$ & $74(50)$ & $10(5.7)$ & $<0.001$ & 0.539 \\
\hline Orbital pain & 0 & $17(11.5)$ & $2(1.1)$ & $<0.001$ & 0.062 \\
\hline Rashes & $5(0.9)$ & $88(59.5)$ & 0 & $<0.001$ & 0.196 \\
\hline Dyspnea & $41(7.8)$ & $3(2)$ & $1(0.6)$ & 0.008 & $<0.001$ \\
\hline Drowsiness & $13(2.5)$ & 0 & 0 & 0.083 & 0.036 \\
\hline Seizures & $8(1.5)$ & 0 & 0 & 0.211 & 0.211 \\
\hline \multicolumn{6}{|l|}{ Laboratory characteristics } \\
\hline Leukopenia (WBC $<3.0 \times 10^{9}$ cells/L) (n/N [\%]) & 9/283 (3.2) & $82 / 146(56)$ & $4 / 152(2.6)$ & $<0.001$ & 0.749 \\
\hline Platelet count $149-100 \times 10^{9}$ cells/L (n/N [\%]) & $38 / 238(15.9)$ & $27 / 146(18.5)$ & $5 / 133(3.7)$ & 0.522 & 0.003 \\
\hline Platelet count $<100 \times 10^{9}$ cells/L (n/N [\%]) & $7 / 238(2.9)$ & $109 / 146(74.6)$ & $3 / 133(2.3)$ & $<0.001$ & 0.696 \\
\hline
\end{tabular}

$\mathrm{WBC}=$ white blood cell count; $\mathrm{n} / \mathrm{N}=$ number of patients/total number of patients with data available.

anfluenza vs. dengue.

${ }^{\mathrm{b}}$ Influenza vs. other febrile illnesses.

${ }^{\mathrm{C}}$ An individual patient might have more than one symptom/sign.

dengue diagnostic test. Of laboratory-positive dengue cases, 40 were confirmed by RT-PCR, 71 by IgM of acute phase serum, 47 by fourfold increase in IgG titer in paired acute and convalescent serum, and 78 by detection of NS1 antigen. Of 71 patients positive for IgM antibody, a fourfold rise in IgG titer in paired serum was

Table 3 Complications of influenza and dengue infections

\begin{tabular}{llll}
\hline Complication $^{\mathrm{a}}$ & $\begin{array}{l}\text { Influenza } \\
(\mathbf{n}=\mathbf{5 2 6})\end{array}$ & $\begin{array}{l}\text { Dengue } \\
(\mathbf{n}=\mathbf{1 4 8})\end{array}$ & $\mathbf{P}$ \\
\hline Pneumonia & $2^{\mathrm{b}}(5.1)$ & $1(0.6)$ & 0.016 \\
ARDS & $5^{\mathrm{c}}(0.9)$ & 0 & 0.234 \\
Meningoencephalitis & $6^{\mathrm{d}}(1.1)$ & 0 & 0.192 \\
Intracranial bleeding & $2^{\mathrm{d}}(0.4)$ & 0 & 0.452 \\
Gastrointestinal bleeding & $2^{\mathrm{d}}(0.4)$ & $14(9.5)$ & $<0.001$ \\
Myocarditis & $1^{\mathrm{d}}(0.2)$ & 0 & 0.596 \\
Acute hepatitis (ALT > 1,000 U/L) & $2^{\mathrm{d}}(0.4)$ & 0 & 0.452 \\
\hline
\end{tabular}

Data are number (\%) of patients. ARDS = acute respiratory distress syndrome; $\mathrm{ALT}=$ alanine aminotransferase.

${ }^{a}$ An individual patient might have more than one complication.

${ }^{b}$ Of 27 influenza patients with pneumonia, 15 cases were 2009 pandemic $\mathrm{H} 1 \mathrm{~N} 1$ and 12 cases were $\mathrm{H} 3 \mathrm{~N} 2$.

'Of 5 influenza patients with ARDS, 4 cases were 2009 pandemic $\mathrm{H} 1 \mathrm{~N} 1$ and 1 case was H3N2.

${ }^{\text {d}}$ Patient(s) with 2009 pandemic H1N1 infection. found in 31 patients, detection of NS1 antigen in 29, positive RT-PCR and NS1 antigen in 6, fourfold increase in IgG titer in paired serum and positive NS1 antigen in 3 , and fourfold rise in IgG titer in paired serum and positive RT-PCR in 2 . The mean time lapse from onset of symptoms to ER presentation was $3.7 \pm 1.8$ days, and the mean fever duration was $4.0 \pm 2.3$ days. The 3 most common symptoms other than fever in dengue patients were malaise $(64.2 \%)$, rashes $(59.5 \%)$, and joint pain (50\%). Marked thrombocytopenia and leukopenia were found in 109 (74.6\%) and 82 (56\%) patients, respectively, of 146 patients with complete blood counts. Forty patients had available RT-PCR data; of these, dengue virus serotype II was detected in 17 (42.5\%) patients, serotype III in $12(30 \%)$, serotype I in 10 (25\%), and serotype IV in $1(2.5 \%)$. Of 148 dengue patients, 14 (9.5\%) experienced gastrointestinal bleeding (Table 3). All dengue patients recovered.

Table 4 describes the diagnosis of the 175 patients with OFI (130 [74.3\%] patients aged $\leq 18$ years). The 3 leading etiologies of OFI were acute pharyngitis (38.8\%), acute bronchitis (25.7\%), and bronchopneumonia (16\%). The mean interval from onset of illness to ER presentation was $3.0 \pm 2.1$ days. All patients with OFI recovered. 


\begin{tabular}{ll} 
Table $\mathbf{4}$ Etiologies of $\mathbf{1 7 5}$ patients with other febrile \\
illnesses \\
\hline Variable & $68(38.8)$ \\
\hline Acute pharyngitis & $45(25.7)$ \\
Acute bronchitis & $28(16)$ \\
Bronchopneumonia & $16(9.1)$ \\
Acute tonsillitis & $11(6.3)$ \\
Gastroenteritis & $2(1.1)$ \\
Sinusitis & $1(0.5)$ \\
Kawasaki & $4(2.3)$ \\
Unknown
\end{tabular}

Data are number (\%) of patients.

\section{Clinical and laboratory characteristics distinguishing influenza from dengue}

Comparisons between patients with influenza $(\mathrm{n}=526)$ and dengue $(\mathrm{n}=148)$ are shown in Tables $1,2,3,5$, and 6. Patients with influenza were significantly younger, had shorter fever duration, and presented at the ER earlier than patients with dengue (Table 1). Rhinorrhea, cough, sore throat, and dyspnea were reported significantly more frequently in patients with influenza than those with dengue (Table 2). Significantly lower frequencies of malaise, headache, abdominal pain, joint pain, orbital pain, and rashes were noted in patients with influenza than in those with dengue (Table 2). Dengue patients had significantly higher incidences of leukopenia, marked thrombocytopenia, and gastrointestinal bleeding in addition to lower incidence of pneumonia (Tables 2 and 3). Multivariate analysis disclosed absence of rashes (odds ratio $[\mathrm{OR}], 131.336$ ), no leukopenia (OR, 24.978), and no marked thrombocytopenia (OR, 105.973) as predictive factors that distinguished influenza from dengue (Table 6). The sensitivities of absence of rashes, without leukopenia, and no marked thrombocytopenia were $98.3 \%, 96.9 \%$, and $97.9 \%$, while the specificities were $60 \%, 55.9 \%$, and $74.5 \%$, respectively.

Symptoms and laboratory characteristics of influenza infection varied by patient age (Table 5). Pediatric influenza patients ( $\leq 18$ years, $n=445$ ) had significantly higher frequencies of rhinorrhea and cough; lower frequencies of malaise, orbital pain and rashes; and lower incidences of leukopenia and marked thrombocytopenia compared with dengue patients $(\mathrm{n}=24)$ (Table 5). Multivariate analysis showed absence of rashes (OR, 326.393), no leukopenia (OR, 122.116) and no marked thrombocytopenia (OR, 88.632) to be independent predictors for distinguishing influenza from dengue in pediatric patients (Table 6). For individuals $>18$ years of age, patients with influenza ( $\mathrm{n}=81$ ) had greater occurrence of rhinorrhea, cough, sore throat, and dyspnea; they also presented with lower frequencies of nausea and vomiting, abdominal pain, joint pain, orbital pain, and rashes as well as lower incidences of leukopenia and marked thrombocytopenia (Table 5) compared to patients with dengue $(\mathrm{n}=124)$. Multivariate analysis indicated that no leukopenia (OR, 13.99) and no marked thrombocytopenia (OR, 34.096) distinguished influenza from dengue in adults (Table 6).

\section{Clinical and laboratory characteristics distinguishing influenza from OFI}

Comparisons between patients with influenza $(n=526)$ and OFI $(n=175)$ are summarized in Tables $1,2,5$, and 6. Significant differences in clinical and laboratory features between influenza and OFI included the presence of rhinorrhea, sore throat, malaise, headache, dyspnea, drowsiness, and mild thrombocytopenia (Table 2). Multivariate analysis revealed that rhinorrhea (OR, 3.350), malaise (OR, 6.050), sore throat (OR, 3.407), dyspnea (OR, 47.335), and mild thrombocytopenia (OR, 3.779) were independent predictive factors that distinguished influenza from OFI (Table 6). The sensitivities of rhinorrhea, malaise, sore throat, dyspnea, and mild thrombocytopenia were $63.9 \%, 44.1 \%, 44.1 \%, 17.3 \%$, and $15.9 \%$, respectively.

As shown in Tables 5 and 6, significantly higher proportions of pediatric patients with influenza $(n=445)$ experienced rhinorrhea, sore throat, malaise, headache, dyspnea, and mild thrombocytopenia compared with OFI ( $\mathrm{n}=130)$; multivariate analysis showed rhinorrhea (OR, 2.765), malaise (OR, 11.129), sore throat (OR, 3.575), dyspnea (OR, 20.867), and mild thrombocytopenia (OR, 7.138 ) to be independent predictive factors that differentiated influenza from OFI in pediatric patients. Compared to adults with OFI $(\mathrm{n}=45)$, adult patients with influenza $(\mathrm{n}=81)$ had significantly higher frequencies of rhinorrhea, cough, malaise, diarrhea, and dyspnea; multivariate analysis revealed rhinorrhea $(\mathrm{OR}, 4.726)$ and malaise $(\mathrm{OR}, 3.108)$ to be predictive factors that distinguished influenza from OFI in adults.

\section{Classification tree distinguishing influenza from dengue}

Variables found to be significant in multivariate logistic regression were used to establish classification and regression trees. As shown in Figure 1, the initial splitting variable in the tree is a rash, followed by leukopenia and marked thrombocytopenia to distinguish influenza from dengue. The three nodes with low probability of influenza infection in pediatric patients were (i) presence of rashes, (ii) absence of rashes but with leukopenia and (iii) absence of rashes, without leukopenia, but with marked thrombocytopenia. There was a high probability of influenza infection among pediatric patients without rashes, no leukopenia and with no marked thrombocytopenia (94.2\% of patients with influenza). Among adult patients, high probability of influenza was associated 
Table 5 Age-specific symptoms/signs and laboratory features of patients with influenza, dengue, and other febrile illnesses

\begin{tabular}{|c|c|c|c|c|}
\hline \multirow[t]{2}{*}{ Variable } & \multicolumn{2}{|l|}{ Age $\leq 18$ years } & \multicolumn{2}{|l|}{ Age $>18$ years } \\
\hline & $\begin{array}{l}\text { Inf vs. Den (Inf, } n=445 \\
\text { Den, } n=24)\end{array}$ & $\begin{array}{l}\text { Inf vs. OFI (Inf, } n=445 \\
\text { OFI, } n=130)\end{array}$ & $\begin{array}{l}\text { Inf vs. Den (Inf, } n=81 \\
\text { Den, } n=124)\end{array}$ & $\begin{array}{l}\text { Inf vs. OFI (Inf, } n=81 \text {; } \\
\text { OFI, } n=45)\end{array}$ \\
\hline \multicolumn{5}{|l|}{ Symptom/sign ${ }^{\text {a }}$ (n [\%]) } \\
\hline Rhinorrhea & $297(66.7)$ vs. $0^{* * *}$ & $297(66.7)$ vs. $65(50)^{* *}$ & $36(44.4)$ vs. $0^{* * *}$ & $36(44.4)$ vs. $8(17.8)^{* *}$ \\
\hline Cough & $311(69.9)$ vs. $3(12.5)^{* * *}$ & 311 (69.9) vs. 94 (72.3) & $45(55.6)$ vs. $35(28.2)^{* * *}$ & $45(55.6)$ vs. $18(40)^{*}$ \\
\hline Sore throat & 163 (36.6) vs. 4 (16.7) & $163(36.6)$ vs. $27(20.8)^{* *}$ & $44(54.3)$ vs. $20(16.1)^{* * *}$ & 44 (54.3) vs. 16 (35.6) \\
\hline Malaise & $141(31.6)$ vs. $14(58.3)^{*}$ & 141 (31.6) vs. $8(6.2)^{* * *}$ & 42 (51.9) vs. 81 (65.3) & $42(51.9)$ vs. $13(28.9)^{*}$ \\
\hline Headache & 125 (28.1) vs. 11 (45.8) & $125(28.1)$ vs. $21(16.2)^{* *}$ & 24 (29.6) vs. 51 (41.1) & 24 (29.6) vs. 12 (26.7) \\
\hline Vomiting/nausea & 106 (23.8) vs. 8 (33.3) & 106 (23.8) vs. 33 (25.4) & $10(12.3)$ vs. $35(28.2)^{* *}$ & 10 (12.3) vs. 4 (8.9) \\
\hline Diarrhea & 54 (12.1) vs. 3 (12.5) & 54 (12.1) vs. 19 (14.6) & 3 (3.7) vs. 12 (9.7) & $3(3.7)$ vs. $7(15.6)^{*}$ \\
\hline Abdominal pain & 34 (7.6) vs. 4 (16.7) & 34 (7.6) vs. 14 (10.8) & $6(7.4)$ vs. $27(21.8)^{* *}$ & 6 (7.4) vs. 4 (8.9) \\
\hline Joint pain & 18 (4) vs. 6 (25) & 18 (4) vs. 7 (5.4) & $8(9.9)$ vs. $68(54.8)^{* * *}$ & 8 (9.9) vs. 3 (6.7) \\
\hline Orbital pain & 0 vs. $2(8.3)^{* *}$ & 0 (0) vs. 2 (1.5) & 0 vs. $15(12.1)^{* *}$ & 0 vs. 0 \\
\hline Rashes & 5 (1.1) vs. $11(45.8)^{* * *}$ & 5 (1.1) vs. 0 & 0 vs. $77(62.1)^{* * *}$ & 0 vs. 0 \\
\hline Dyspnea & 24 (5.6) vs. 1 (4.1) & $24(5.6)$ vs. $1(0.8)^{*}$ & $17(3.7)$ vs. $2(1.6)^{* * *}$ & $17(3.7)$ vs. $0^{* *}$ \\
\hline Drowsiness & $10(2.2)$ vs. 0 & $10(2.2)$ vs. 0 & 3 (3.7) vs. 0 & 3 (3.7) vs. 0 \\
\hline Seizures & 6 (1.3) vs. 0 & $6(1.3)$ vs. 0 & $2(2.5)$ vs. 0 & $2(2.5)$ vs. 0 \\
\hline \multicolumn{5}{|l|}{ Laboratory characteristics } \\
\hline $\begin{array}{l}\text { Leukopenia }\left(\mathrm{WBC}<3.0 \times 10^{9} \text { cells/L) }\right. \\
(\mathrm{n} / \mathrm{N}[\%])\end{array}$ & $6 / 231(2.6)$ vs. $14 / 23(60.8)^{* * *}$ & $6 / 231$ (2.6) vs. $1 / 116(0.8)$ & $3 / 52(5.8)$ vs. $68 / 123(55.3)^{* * *}$ & $3 / 52$ (5.8) vs. $3 / 36(8.3)$ \\
\hline $\begin{array}{l}\text { Platelet count } 149-100 \times 10^{9} \text { cells/L } \\
(\mathrm{n} / \mathrm{N}[\%])\end{array}$ & $24 / 189$ (12.6) vs. $6 / 23$ (26) & $24 / 189(12.6)$ vs. $2 / 106(1.9)^{* *}$ & $14 / 49$ (28.6) vs. $21 / 123$ (17.1) & $14 / 49$ (28.6) vs. $3 / 27$ (11.1) \\
\hline $\begin{array}{l}\text { Platelet count }<100 \times 10^{9} \text { cells } / \mathrm{L} \\
(\mathrm{n} / \mathrm{N}[\%])\end{array}$ & $3 / 189(1.6)$ vs. $12 / 23(52.2)^{* * *}$ & 3/189 (1.6) vs. 2/106 (1.9) & 4/49 (8.2) vs. $97 / 123(78.9)^{* * *}$ & 4/49 (8.2) vs. $1 / 27$ (3.7) \\
\hline
\end{tabular}

Inf = influenza; Den = dengue; $\mathrm{OFI}=$ other febrile illnesses; $\mathrm{WBC}=$ white blood count; $\mathrm{n} / \mathrm{N}=$ number of patients/total number of patients with data available. ${ }^{*} \mathrm{P}<0.05$; ${ }^{* *} \mathrm{P}<0.01$; ${ }^{* * *} \mathrm{P}<0.001$.

${ }^{\mathrm{a}}$ An individual patient might have more than one symptom/sign.

with no leukopenia and no marked thrombocytopenia (87.8\% of patients with influenza) (Figure 2).

\section{Discussion}

Our study investigated simple clinical and laboratory features to identify patients with influenza among children and adults with acute febrile illness in ER in dengue and non-dengue endemic areas. In our series, the mean time lapse between onset of illness and ER presentation was 1.7, 3.7, and 3 days for influenza, dengue, and OFI, respectively. This permits a detailed comparison of early clinical and laboratory characteristics between influenza and dengue or OFI.

The symptoms of influenza overlap substantially with dengue and OFI (Table 2). We found that symptoms resulting from respiratory tract infections such as cough, rhinorrhea, sore throat, and dyspnea were more prevalent in patients with influenza than with dengue. In contrast, non-respiratory tract symptoms including rashes, headache, abdominal pain, joint pain, and orbital pain were less common in the patients with influenza compared to patients with dengue. The influenza virus primarily infects epithelial cells of the respiratory tract and causes upper respiratory symptoms [25]. Damage to infected cells results in release of inflammatory mediators, leading to a systemic response (i.e., fever, headache, joint pain, malaise, and myalgia) with symptoms similar to other viral illnesses such as dengue [26,27].

The overlapping geographic range of influenza and dengue as well as simultaneous dengue and influenza outbreaks [8-11], often in resource-limited countries, leads to diagnostic difficulties, as nonspecific symptoms are common to both infections. Early detection of influenza and dengue is especially important because influenza may be prevented through proper isolation and treated using antiviral agents $[28,29]$, whereas failure to make a timely dengue diagnosis with adequate fluid replacement can lead to severe dengue [30]. Our study demonstrated that the presence of leukopenia (white cell count $<3.0 \times 10^{9}$ cells/L) and marked thrombocytopenia (platelet count $<100 \times 10^{9}$ cells/L) are useful for differentiating dengue from influenza in both adults and pediatric 
Table 6 Sensitivity, specificity, and multivariate logistic regression for prediction of influenza versus dengue and influenza versus other febrile illnesses

\begin{tabular}{|c|c|c|c|c|c|c|c|c|}
\hline & $\begin{array}{l}\text { Influenza } \\
\text { n/N (\%) }\end{array}$ & $\begin{array}{l}\text { Dengue } \\
\mathrm{n} / \mathrm{N}(\%)\end{array}$ & $\begin{array}{l}\text { Other febrile } \\
\text { illnesses } \mathrm{n} / \mathrm{N}(\%)\end{array}$ & $\begin{array}{l}\text { Adjusted odds } \\
\text { ratio }\end{array}$ & $95 \% \mathrm{Cl}$ & $\mathbf{P}$ & Sensitivity\% & Specificity\% \\
\hline \multicolumn{9}{|l|}{ Influenza vs. dengue } \\
\hline \multicolumn{9}{|l|}{ All ages } \\
\hline No skin rashes & $234 / 238(98.3)$ & $58 / 145(40)$ & - & 131.336 & $35.416-487.039$ & $<0.001$ & 98.3 & 60 \\
\hline No leukopenia ${ }^{a}$ & 225/232 (96.9) & $64 / 145(44.1)$ & - & 24.978 & $7.010-89.006$ & $<0.001$ & 96.9 & 55.9 \\
\hline No marked thrombocytopenia ${ }^{b}$ & 233/238 (97.9) & $37 / 145(25.5)$ & - & 105.973 & $31.687-354.419$ & $<0.001$ & 97.9 & 74.5 \\
\hline \multicolumn{9}{|l|}{ Age $\leq 18$ years } \\
\hline No skin rashes & 185/189 (97.9) & $12 / 23(52.2)$ & - & 326.393 & $30.860-3452.098$ & $<0.001$ & 97.9 & 52.2 \\
\hline No leukopenia ${ }^{a}$ & 185/189 (97.9) & 9/23 (39.1) & - & 122.116 & $10.888-1369.665$ & $<0.001$ & 97.9 & 60.9 \\
\hline No marked thrombocytopenia ${ }^{\text {b }}$ & 186/189 (98.4) & $11 / 23(47.8)$ & - & 88.632 & 6.443-1219.174 & 0.001 & 98.4 & 52.2 \\
\hline \multicolumn{9}{|l|}{ Age $>18$ years } \\
\hline No leukopenia ${ }^{a}$ & 46/49 (93.9) & $55 / 122(45.1)$ & - & 13.99 & $3.628-53.946$ & $<0.001$ & 93.9 & 54.9 \\
\hline No marked thrombocytopenia ${ }^{b}$ & $45 / 49(91.8)$ & $26 / 122(21.3)$ & - & 34.096 & 10.622-109.445 & $<0.001$ & 91.8 & 78.7 \\
\hline \multicolumn{9}{|l|}{$\begin{array}{l}\text { Influenza vs. other febrile } \\
\text { illnesses }\end{array}$} \\
\hline \multicolumn{9}{|l|}{ All ages } \\
\hline Rhinorrhea & $152 / 238(63.9)$ & - & $57 / 137(41.6)$ & 3.350 & $1.997-5.618$ & $<0.001$ & 63.9 & 58.4 \\
\hline Malaise & $105 / 238(44.1)$ & - & $16 / 137(11.7)$ & 6.050 & $3.207-11.414$ & $<0.001$ & 44.1 & 88.3 \\
\hline Sore throat & 105/238 (44.1) & - & 30/137 (21.9) & 3.407 & $1.960-5.922$ & $<0.001$ & 44.1 & 78.1 \\
\hline Dyspnea & $41 / 238(17.3)$ & - & $1 / 137(0.7)$ & 47.335 & $6.174-632.919$ & $<0.001$ & 17.3 & 99.3 \\
\hline Mild thrombocytopeniac ${ }^{c}$ & $38 / 238(15.9)$ & - & 5/137 (3.6) & 3.779 & $1.309-10.908$ & 0.014 & 15.9 & 96.4 \\
\hline \multicolumn{9}{|l|}{ Age $\leq 18$ years } \\
\hline Rhinorrhea & 131/189 (69.3) & - & $53 / 106(50)$ & 2.765 & $1.531-4.997$ & 0.001 & 69.3 & 50 \\
\hline Malaise & $76 / 189(40.2)$ & - & $6 / 106(5.7)$ & 11.129 & $4.455-27.802$ & $<0.001$ & 40.2 & 94.3 \\
\hline Sore throat & 83/189 (43.9) & - & 21/106 (19.8) & 3.575 & $1.892-6.753$ & $<0.001$ & 43.9 & 80.2 \\
\hline Dyspnea & 24/189 (12.7) & - & $1 / 106(0.9)$ & 20.867 & $2.641-164.872$ & 0.004 & 12.7 & 99 \\
\hline Mild thrombocytopeniac & $24 / 189(12.7)$ & - & 2/106 (1.9) & 7.138 & $1.509-33.769$ & 0.013 & 12.7 & 98.1 \\
\hline Age $>18$ years & & - & & & & & & \\
\hline Rhinorrhea & $36 / 81(44.4)$ & - & $8 / 45(17.8)$ & 4.726 & $1.654-13.5$ & 0.004 & 44.4 & 82.2 \\
\hline Malaise & $42 / 81(51.9)$ & - & 13/45 (28.9) & 3.108 & $1.224-7.897$ & 0.017 & 51.9 & 71.1 \\
\hline
\end{tabular}

$\mathrm{n} / \mathrm{N}=$ number of patients/total number of patients with data available; $\mathrm{Cl}=$ confidence interval.

a Leukopenia is defined as white cell counts $<3.0 \times 10^{9}$ cells $/ \mathrm{L}$.

${ }^{\mathrm{b}}$ Marked thrombocytopenia is defined as platelet counts $<100 \times 10^{9}$ cells/L.

${ }^{c}$ Mild thrombocytopenia is defined as platelet counts $149-100 \times 10^{9}$ cells/L.

patients. The absence of rash further discriminated influenza from dengue in pediatric patients. Rash associated with influenza is not a common manifestation, occurring in only $2-8 \%$ of patients, usually children [31,32]. In contrast, the reported frequency of rash in dengue cases ranges from 46-68\%, particularly in children less than 15 years who usually have a nonspecific febrile syndrome accompanied by rash [33,34]. Mild leukopenia and relative lymphopenia are typical findings of influenza; thrombocytopenia may be present in complicated cases $[4,14,25]$. Notably, our study and others found leukopenia and thrombocytopenia to be common laboratory findings in dengue infection, with platelet counts below $20 \times 10^{9}$ per liter often observed in severe dengue [7,30]. A study examining predictors of diagnosis in 1,962 febrile travelers returning from the tropics found the main predictors of dengue infection compared with other fevers, excluding malaria, to include skin rash, thrombocytopenia, and leukopenia [35]. This finding was consistent with our study findings.

Our data suggest that skin rash, leukopenia (white cell count $<3.0 \times 10^{9}$ cells/L), and platelet counts $<100 \times 10^{9}$ cells/L were useful to predict negative influenza results during influenza and dengue epidemics. However, it is 


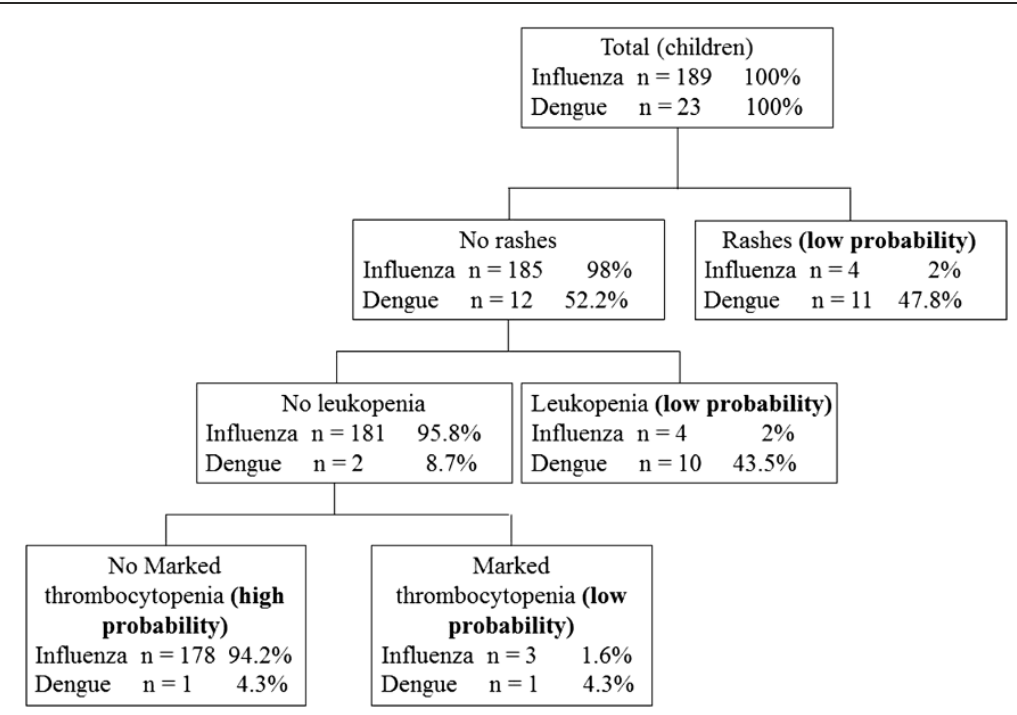

Figure 1 Diagnostic algorithm to discriminate between influenza and dengue in pediatric patients. Terminal nodes are marked as "low probability" or "high probability" for influenza infection.

unlikely that any single indicator will be useful in clinical practice because these symptoms and laboratory findings can be present in both infections [11]. In our series, we developed two simple and practical diagnostic algorithms using clinical and laboratory indicators to distinguish influenza from dengue in adult and pediatric patients, respectively. We found that the diagnostic algorithm correctly classified $94.2 \%$ of pediatric patients with influenza in the "high probability" group with only one misclassified dengue patient (Figure 1). Analysis of our data after excluding pediatric patients showed similar results, except that skin rash that was no longer associated with influenza infection in adult patients; however, the diagnostic tree (Figure 2) still correctly classified $87.8 \%$ of adult influenza cases as "high probability". These findings underscored that rash, leukopenia, and marked thrombocytopenia could help to establish a diagnostic algorithm to distinguish influenza from dengue patients during outbreaks of both diseases. Additional prospective studies are needed to validate this predictive model in other dengue-endemic regions and in populations with different ethnicities.

The wide range of influenza-associated symptoms often makes it difficult to distinguish from other febrile

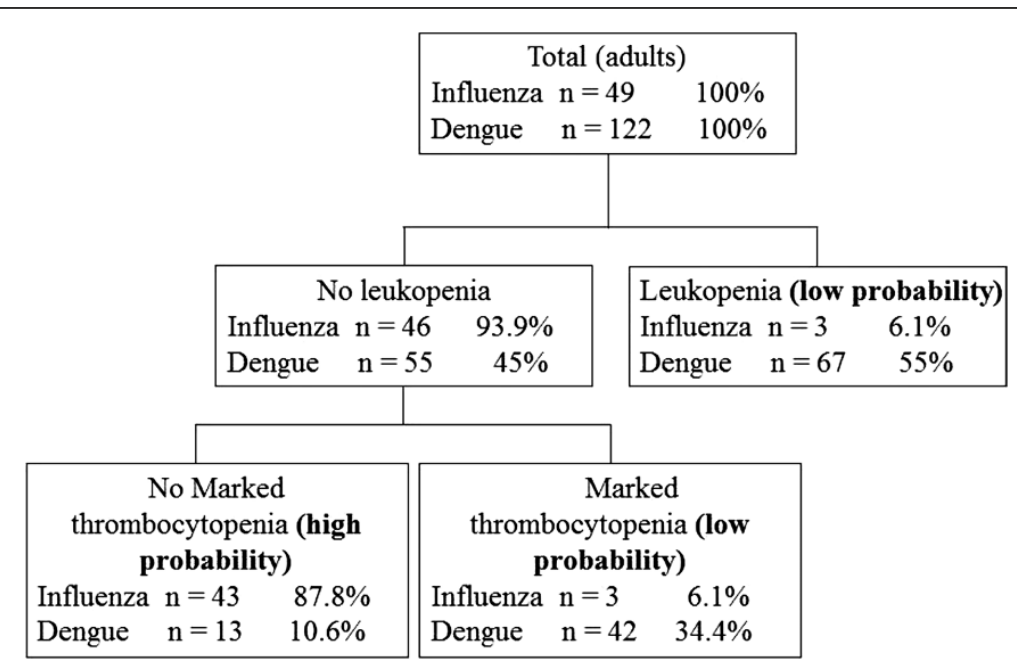

Figure 2 Diagnostic algorithm to discriminate between influenza and dengue in adult patients. Terminal nodes are marked as "low probability" or "high probability" for influenza infection. 
or respiratory illnesses [25]. A crowded ER can make it challenging for physicians to differentiate between influenza and OFI [36]. Early antiviral treatment ( $\leq 48 \mathrm{~h})$ of influenza is especially important for patients with underlying risk factors to avoid otherwise preventable morbidity and mortality [37]. Our results demonstrate that rhinorrhea, malaise, sore throat, and dyspnea in addition to a slightly low platelet count (100-149 $\times 10^{9}$ cells/L) in children, as well as rhinorrhea and malaise in adults were valuable predictors during the evaluation of the likelihood of influenza versus OFI in a non-dengue endemic setting. However, apart from rhinorrhea, which lacked adequate sensitivity and specificity, we found that all other variables were specific (>70\%) but not sensitive enough in distinguishing influenza from OFI. This is not surprising as more than $60 \%$ of OFI cases included in this series were individuals with pharyngitis or bronchitis (Table 4), another viral infection commonly encountered in the ER [38]. The information of this study are most helpful for clinicians to facilitate diagnosis of influenza during periods of high influenza activity in non-dengue endemic setting.

In the present study, patients with influenza had a shorter fever duration than the dengue cases (mean $2.8 \pm 1.6$ vs. $4.0 \pm 2.3$ days). This finding is similar to a previous study that found a fever duration of $<4$ days for patients with influenza [25], while the average length of fever in dengue patients was approximately 5 days [39], coinciding with the disappearance of viremia.

Although influenza primarily causes upper respiratory tract infections, pulmonary and extra-pulmonary complications have also been reported [2,40,41]. Previous studies from the United States and Australia of critically ill patients with 2009 pandemic H1N1 infections found ARDS complication in $35.8 \%$ and $48.8 \%$ of cases and a $45 \%$ and $14.3 \%$ hospital mortality, respectively [40,41]. ARDS was noted in 5 influenza cases in our study (Table 3); 3 were fatal. Remarkably, delayed oseltamivir therapy was found in all fatal cases in our series. The importance of a timely anti-viral therapy for severe influenza should therefore be emphasized.

Earlier studies describe neurological complications of influenza including aseptic meningitis, encephalopathy/ encephalitis, Guillain-Barré syndrome, and transverse myelitis [42-44]. Neurological complications have been reported for patients with 2009 pandemic H1N1 infections [43]. Of 447 patients with 2009 pandemic H1N1 infection in this study, meningoencephalitis was found in $6(1.3 \%)$, with presenting symptoms of altered mental status and seizures (Table 3). Other influenza-associated extrapulmonary complications among our patients included myocarditis, rhabdomyolysis, gastrointestinal bleeding, and intracranial hemorrhage (Table 3). Physicians should be aware of influenza-associated extrapulmonary complications when caring for influenza patients and manage them accordingly.

Gastrointestinal bleeding was the most common complication among dengue patients in this study. Previous studies have reported gastrointestinal bleeding to be a warning sign of severe dengue; timely management and intensive monitoring of patients with gastrointestinal bleeding is therefore necessary [45].

This study has several limitations. First, because it was conducted in a single medical center, disease severity may have been biased by referral patterns. Second, as a retrospective study, missing laboratory data was inevitable. Third, the decision to perform diagnostic tests for influenza and dengue infections was based on individual physicians' experience; therefore, patients included in our series may have been biased by individual physicians' personal judgments. In addition, our results were based on data from early stages of illness; future studies are necessary to validate these findings in different course of illness for better generalization of their utility.

\section{Conclusions}

This study demonstrates substantial overlap in clinical presentation between influenza and dengue as well as OFI. This study also identifies simple and useful clinical and laboratory data to enable identification and facilitate diagnosis of influenza in different clinical settings (dengue and non-dengue endemic areas). We provide two decision tree algorithms using simple clinical and laboratory data that can be easily implemented in resource-limited countries to differentiate patients with influenza from those with dengue. This information is especially important to clinicians in countries where medical resources are sparse and the burden of influenza and dengue is high.

\section{Competing interests}

The authors declare that they have no competing interests.

\section{Authors' contributions}

SYH and IKL made major contributions to sample collection and wrote the manuscript. IKL conceived and designed the study, analysis of the data and preparation of the manuscript. SYH, SCH, CCC, TYC, WCH, JWL and LW collected clinical data. All authors read, commented on, and approved the final manuscript.

\section{Author details}

${ }^{1}$ Department of Emergency Medicine, Kaohsiung Chang Gung Memorial Hospital, Kaohsiung 833, Taiwan. ²Division of Infectious Diseases, Department of Internal Medicine, Kaohsiung Chang Gung Memorial Hospital, Kaohsiung 833, Taiwan. ${ }^{3}$ Department of Pediatrics, Kaohsiung Chang Gung Memorial Hospital, Kaohsiung 833, Taiwan.

Received: 16 November 2013 Accepted: 7 November 2014 Published online: 25 November 2014

\section{References}

1. Briand S, Mounts A, Chamberland M: Challenges of global surveillance during an influenza pandemic. Public Health 2011, 125(5):247-256 
2. Rothberg MB, Haessler SD, Brown RB: Complications of viral influenza. Am J Med 2008, 121(4):258-264.

3. Murata $Y$, Walsh EE, Falsey AR: Pulmonary complications of interpandemic influenza A in hospitalized adult. J Infect Dis 2007, 195(7):1029-1037.

4. Cate TR: Clinical manifestations and consequences of influenza. Am J Med 1987, 82(6A):15-19.

5. Guzmán MG, Kourí G: Dengue: an update. Lancet Infect Dis 2002, 2(1):33-42.

6. Tsai CY, Lee IK, Lee CH, Yang KD, Liu JW: Comparisons of dengue illness classified based on the 1997 and 2009 World Health Organization dengue classification schemes. J Microbiol Immunol Infect 2013, 46(4):271-281.

7. World Health Organization: Dengue Hemorrhagic Fever: Diagnosis, Treatment and Control. Geneva: World Health Organization; 2009.

8. Silarug N, Foy HM, Kupradinon S, Rojanasuphot S, Nisalak A, Ponguswant Y: Epidemic of fever of unknown origin in rural Thailand caused by influenza A (H1N1) and dengue fever. Southeast Asian J Trop Med Public Health 1990, 21(1):61-67.

9. Hussain R, Al-Omar I, Memish ZA: The diagnostic challenge of pandemic H1N1 2009 virus in a dengue-endemic region: a case report of combined infection in Jeddah, Kingdom of Saudi Arabia. J Infect Public Health 2012, 5(2):199-202.

10. Lopez Rodriguez E, Tomashek KM, Gregory CJ, Munoz J, Hunsperger E, Lorenzi OD, Irizarry JG, Garcia-Gubern C: Co-infection with dengue virus and pandemic (H1N1) 2009 virus. Emerg Infect Dis 2010, 16(5):882-884.

11. Morens DM, Rigau-Pérez JG, López-Correa RH, Moore CG, Ruiz-Tibén EE, Sather GE, Chiriboga J, Eliason DA, Casta-Velez A, Woodall JP: Dengue in Puerto Rico, 1977: public health response to characterize and control an epidemic of multiple serotypes. Am J Trop Med Hyg 1986, 35(1):197-211.

12. Lee IK, Lee WH, Yang KD, Liu JW: Comparison of the effects of oral hydration and intravenous fluid replacement in adult patients with non-shock dengue hemorrhagic fever in Taiwan. Trans $R$ Soc Trop Med Hyg 2010, 104(8):541-545.

13. Lee IK, Liu JW, Yang KD: Clinical and laboratory characteristics and risk factors for fatality in elderly patients with dengue hemorrhagic fever. Am J Trop Med Hyg 2008, 79(2):149-153.

14. Lee IK, Liu JW, Wang L, Yang KD, Li CC, Eng HL: 2009 pandemic influenza A (H1N1): clinical and laboratory characteristics in pediatric and adult patients and in patients with pulmonary involvement. Influenza Other Respir Viruses 2012, 6(6):e152-161.

15. Cheng CK, Cowling BJ, Chan KH, Fang VJ, Seto WH, Yung R, Uyeki TM, Houck PM, Peiris JS, Leung GM: Factors affecting QuickVue Influenza A+B rapid test performance in the community setting. Diagn Microbiol Infect Dis 2009, 65(1):35-41.

16. Shu PY, Huang JH: Current advances in dengue diagnosis. Clin Diagn Lab Immunol 2004, 11(4):642-650

17. World Health Organization: Clinical management of human infection with pandemic (H1N1) 2009: revised guidance. Available at http://www.who.int/ csr/resources/publications/swineflu/clinical_management/en/index.html (Accessed November 2009).

18. Trademarks: QIAGEN": QIAamp Viral RNA Mini Kit-for purification of viral RNA from cell-free body fluids. Available at http://www.qiagen.com/ MyQIAcube (Accessed November 2007).

19. World Health Organization: $C D C$ protocol of real-time RT-PCR for influenza A (H1N1). Geneva: World Health Organization.

20. Shu PY, Chang SF, Kuo YC, Yueh YY, Chien $\sqcup$, Sue CL, Lin TH, Huang JH: Development of group- and serotype-specific one-step SYBR Green I-based real-time reverse transcription-PCR assay for dengue virus. J Clin Microbiol 2003, 41(6):2408-2416.

21. Shu PY, Chen LK, Chang SF, Yueh YY, Chow L, Chien LJ, Chin C, Lin TH, Huang $\mathrm{JH}$ : Comparison of capture immunoglobulin $M(\mathrm{IgM})$ and $\lg G$ enzyme-linked immunosorbent assay (ELISA) and nonstructural protein NS1 serotype-specific IgG ELISA for differentiation of primary and secondary dengue virus infections. Clin Diagnos Lab Immunol 2003, 10(4):622-630.

22. Shu PY, Yang CF, Kao JF, Su CL, Chang SF, Lin CC, Yang WC, Shih H, Yang SY, Wu PF, Wu HS, Huang JH: Application of the dengue virus NS1 antigen rapid test for onsite detection of imported dengue cases at airports. Clin Vaccine Immunol 2009, 16(4):589e-91.
23. Centers for Disease Control, Department of Health, R.O.C (Taiwan): Guidelines for dengue control. R.O.C (Taiwan): Centers for Disease Control, Department of Health; 2009 [in Chinese].

24. Crichton NJ, Hinde JP, Marchini J: Models for diagnosing chest pain: is CART helpful ? Stat Med 1997, 16(7):717-727.

25. Eccles R: Understanding the symptoms of the common cold and influenza. Lancet Infect Dis 2005, 5(11):718-725.

26. Brydon EW, Morris SJ, Sweet C: Role of apoptosis and cytokines in influenza virus morbidity. FEMS Microbiol Rev 2005, 29(4):837-850.

27. Matsukura S, Kokubu F, Kubo H, Tomita T, Tokunaga H, Kadokura M, Yamamoto T, Kuroiwa Y, Ohno T, Suzaki H, Adachi M: Expression of RANTES by normal airway epithelial cells after influenza virus $\mathrm{A}$ infection. Am J Respir Cell Mol Biol 1998, 18(2):255-264.

28. Jeong I, Lee CH, Kim DK, Chung HS, Park SW: Mild form of 2009 H1N influenza infection detected by active surveillance: implications for infection control. Am J Infect Control 2010, 38(6):482-485.

29. Higuera Iglesias AL, Kudo K, Manabe T, Corcho Berdugo AE, Corrales Baeza A, Alfaro Ramos L, Guevara Gutiérrez R, Manjarrez Zavala ME, Takasaki J, Izumi S, Bautista E, Perez Padilla JR: Reducing occurrence and severity of pneumonia due to pandemic H1N1 2009 by early oseltamivir administration: a retrospective study in Mexico. PLOS One 2011, 6(7):e21838.

30. Simmons CP, Farrar JJ, Nguyen W, Wills B: Dengue. N Engl J Med 2012, 366(15):1423-1432.

31. Hope-Simpson RE, Higgins PG: A respiratory virus study in Great Britain: review and evaluation. Prog Med Virol 1969, 11:354-407.

32. Ryan-Poirier K: Influenza virus infection in children. Adv Pediatr Infect Dis 1995, 10:125-156.

33. Thomas EA, John M, Bhatia A: Cutaneous manifestations of dengue viral infection in Punjab (north India). Int J Dermatol 2007, 46(7):715-719.

34. Saleem K, Shaikh I: Skin lesions in hospitalized cases of dengue Fever. J Coll Physicians Surg Pak 2008, 18(10):608-611.

35. Bottieau E, Clerinx J, Van den Enden E, Van Esbroeck M, Colebunders R, Van Gompel A: Fever after a stay in the tropics: diagnostic predictors of the leading tropical conditions. Medicine (Baltimore) 2007, 86(1):18-25.

36. Monmany J, Rabella N, Margall N, Domingo P, Gich I, Vazquez G: Unmasking influenza virus infection in patients attended to in the emergency department. Infection 2004, 32(2):89-97.

37. Writing Committee of the WHO Consultation on Clinical Aspects of Pandemic (H1N1) 2009 Influenza, Bautista E, Chotpitayasunondh T, Gao Z, Harper SA, Shaw M, Uyeki TM, Zaki SR, Hayden FG, Hui DS, Kettner JD, Kumar A, Lim M, Shindo N, Penn C, Nicholson KG: Clinical aspects of pandemic 2009 influenza A (H1N1) virus infection. N Engl J Med 2010, 362(18):1708-1719.

38. Kumar R, Tripathi P, Tripathi S, Kanodia A, Pant S, Venkatesh V: Prevalence and clinical differentiation of dengue fever in children in northern India. Infection 2008, 36(5):444-449

39. Lee IK, Liu JW, Yang KD: Clinical characteristics and risk factors for concurrent bacteremia in adults with dengue hemorrhagic fever. Am J Trop Med Hyg 2005, 72(2):221-226.

40. Jain S, Kamimoto L, Bramley AM, Schmitz AM, Benoit SR, Louie J, Sugerman DE, Druckenmiller JK, Ritger KA, Chugh R, Jasuja S, Deutscher M, Chen S, Walker JD, Duchin JS, Lett S, Soliva S, Wells EV, Swerdlow D, Uyeki TM, Fiore AE, Olsen SJ, Fry AM, Bridges CB, Finelli L, Pandemic Influenza A (H1N1) Virus Hospitalizations Investigation Team: Hospitalized patients with $2009 \mathrm{H} 1 \mathrm{~N} 1$ influenza in the United States, April-June 2009. N Engl J Med 2009, 361(20):1935-1944.

41. Webb SA, Pettilä V, Seppelt I, Bellomo R, Bailey M, Cooper DJ, Cretikos M, Davies AR, Finfer S, Harrigan PW, Hart GK, Howe B, Iredell JR, McArthur C, Mitchell I, Morrison S, Nichol AD, Paterson DL, Peake S, Richards B, Stephens D, Turner A, Yung M: Critical care services and 2009 H1N1 influenza in Australia and New Zealand. N Engl J Med 2009, 361(20):1925-1934.

42. Hjalmarsson A, Blomqvist $P$, Brytting $M$, Linde A, Skoldenberg B: Encephalitis after influenza in sweden 1987-1998: a rare complication of a common infection. Eur Neuro 2009, 61(5):289-294.

43. Glaser CA, Winter K, DuBray K, Harriman K, Uyeki TM, Sejvar J, Gilliam S, Louie JK: A population-based study of neurologic manifestations of severe influenza A (H1N1) pdm09 in california. Clin Infect Dis 2012, 55(4):514-520. 
44. Sivadon-Tardy V, Orlikowski D, Porcher R, Sharshar T, Durand MC, Enouf V, Rozenberg F, Caudie C, Annane D, van der Werf S, Lebon P, Raphaël JC, Gaillard JL, Gault E: Guillain-barre syndrome and influenza virus infection. Clin Infect Dis 2009, 48(1):48-56.

45. Lee IK, Liu JW, Yang KD: Fatal dengue hemorrhagic fever in adults: emphasizing the evolutionary pre-fatal clinical and laboratory manifestations. PLoS Negl Trop Dis 2012, 6(2):e1532.

doi:10.1186/s12879-014-0623-z

Cite this article as: Huang et al:: Use of simple clinical and laboratory

predictors to differentiate influenza from dengue and other febrile

illnesses in the emergency room. BMC Infectious Diseases 2014 14:623.

\section{Submit your next manuscript to BioMed Central and take full advantage of:}

- Convenient online submission

- Thorough peer review

- No space constraints or color figure charges

- Immediate publication on acceptance

- Inclusion in PubMed, CAS, Scopus and Google Scholar

- Research which is freely available for redistribution 